Background: Radioactive contamination can arise from accidents involving nuclear reactors, industrial sources, or medical sources. Uncontrolled chain reaction during nuclear reactor accident or nuclear bomb explosion results in the release of a number of radionuclides, especially the long-lived radioisotopes. Methods: The Phase Inversion Temperature (PIT) method was employed for the preparation of nanoemulsion. Prepared nanoemulsion was found to be uniformly homogenous and stable. Dynamic Light Scattering (DLS) measurements were carried out using Nano-sizer/Zetasizer ZS (HORIBA La-900, UK). Globule size of the nanoemulsion is in the expected nano-range. The globules of nanoemulsion are in the expected nano-range, as determined by using the Transmission Electron microscope (Morgagni 268D, FEI, Holland). Te homogeneous phase of the nanoemulsion was determined by acquiring confocal microscopic images of the Rhodamine 123-treated nanoemulsion with an optical Leitz Confocal microscope (Leica TCS SP2 UV, Wetzlar, Germany) equipped with Coolsnap ES camera (Roper Scientific, Evry, France).

Results: Before and after each decontamination attempt, whole body counts were recorded with $\mathrm{NaI}(\mathrm{Tl})$ detectors mounted on chair geometry. The 1,026 channel acquisition time was kept as 10 minutes. A significant decrease in the radioactivity were recorded for $99 \mathrm{mTc}$, 131I \& 201Tl. The results obtained comply with the previously published results.

Conclusion: Developed nanoemulsion could be effectively used for decontamination of the radioisotopes from skin. To remove most of the contaminants, only one to two decontamination attempts are enough. Radioactive waste generation could also be limited. These studies show that the nanoemulsion of $\mathrm{p}$-tertbutylcalix[4]arene could be used as a decontamination formulation against the broad range of radioactive nuclides.

Prehosp Disaster Med 2017;32(Suppl. 1):s1-s2

doi:10.1017/S1049023X17000334

Let There be Light: Evaluating Decontamination Effectiveness during a Large-scale Simulation of a CBRNe Disaster

Esli Osmanlliu ${ }^{1}$,Elene Khalit ${ }^{2}$, Ilana Bank ${ }^{3}$, Peter Nugus ${ }^{4}$, Margaret Ruddy ${ }^{5}$, Meredith Young ${ }^{6}$

1. Pediatrics, Montreal Children's Hospital, Montreal/QC/Canada,

2. Pediatric Emergency Medicine, Montreal Children's Hospital, Montreal/QC/Canada

3. Pediatric Emergency Medicine, Montreal Children's Hospital, Montreal/Canada

4. Center For Medical Education, McGill University, Montreal/QC/ Canada

5. Pediatric Intensive Care Unit, Montreal Children's Hospital, Montreal/Canada

6. Department Of Medicine, McGill University, Montrea//QC/ Canada

Study/Objective: Evaluate the effectiveness of patient decontamination during a disaster simulation using a visual tool.

Background: Chemical, biological, radiological, nuclear, and explosive (CBRNe) disasters have significant impact on affected populations. Health care workers (HCWs) must be prepared to execute a Disaster Plan in order to mitigate the negative health outcomes of such events. Decontamination constitutes a major component of disaster response. It optimizes health outcomes by limiting the incidence of secondary, contaminant-mediated injury. Maintaining a "locked down" of the decontaminated care area also reduces the risk of significant injury among exposed HCW and uncontaminated patients. This study proposes an objective assessment of decontamination effectiveness, which lacks in the literature.

Methods: We organized the largest documented pediatric, hospital-wide, disaster simulation with 64 simulated patients and $97 \mathrm{HCW}$ participants. After a brief training, participating HCWs executed the decontamination procedure for the first time. Liquid-based Glo Germ ${ }^{\mathrm{TM}}$ was randomly applied on different body areas, and recorded in 30 simulated patients. Using an ultraviolet light, two independent raters evaluated the total contaminated body surface area before and after decontamination. Simulated patients triaged as contaminated went through a sequence of undressing, followed by low-pressure, high-volume water and soap washing. Effectiveness of decontamination was calculated using a prepared standardized diagram of body surface area. Inter-rater reliability was assessed with a two-way, mixed consistency, average-measures, intraclass correlation coefficient (ICC) using SPSS.

Results: Undressing followed by washing led to an average $80.6 \%$ reduction in total body contamination $(95 \%$ CI [73.6-87.6]). The ICC was 0.91 (95\% CI [0.81-0.96]), indicating that decontamination was evaluated similarly between raters.

Conclusion: A liquid-based visual tool, used as a way to determine decontamination efficacy, is easily obtainable and innovative, and it can help establish verifiable decontamination standards in disaster literature. Undressing followed by washing led to an average $80.6 \%$ decrease in total body contamination.

Prehosp Disaster Med 2017;32(Suppl. 1):s2

doi:10.1017/S1049023X17000346

Preparing a Tertiary Medical Center for a "Dirty-Bomb" Threat Dagan Schwartz ${ }^{1}$, Dorit Nagar ${ }^{1}$, Michal Hayat ${ }^{1}$, Tamar Rubinstein ${ }^{2}$

1. Emergency Prepardness, Rabin Medical Center, Petach-Tikva/ Israel

2. Emergency Medicine, Rabin Medical Center, Petach-Tikva/Israel

Study/Objective: To review hospital preparations and drill design, of a tertiary medical center for a "dirty-bomb" scenario. Background: Terror risk in general, and specifically the risk of terror related to a dirty bomb deployment has increased in resent years. Though the radiation injuries expected to occur in such a scenario are minor, in comparison to the conventional injuries, the psychological impact and the resulting area contamination are expected to be significant. The Israeli Ministry of Health guides and evaluates public hospitals preparedness measures, for a variety of conventional and none conventional scenarios; these include radiological threats. In April 2016 following 6 months of preparations, a "dirty-bomb" drill was conducted at the Beilinson tertiary medical center.

Methods: Descriptive analysis of the drill design and the preparatory actions. 
Results: Preparations included training radiation monitoring personnel, decontamination station's staff and training of $\mathrm{ED}$, and ED reinforcement medical and ancillary staff. The main sites that were prepared and later drilled included: The decontamination site in which patients with possible radiologic contamination were decontaminated and received emergency care, The staff radiation clearance stations, The designated ED areas for care of potentially contaminated patients, The uncontaminated ED areas including areas for acute stress reaction victims, The ED imaging facilities and a designated OR for care of contaminated patients requiring surgical decontamination, or other urgent surgeries, in patients of whom routine external decontamination was insufficient. A total of 220 hospital employees participated in formal training sessions, preparatory internal drills and the final full scale drill.

Conclusion: The "dirty-bomb" scenario for a receiving hospital is challenging. It requires identification of radiological contamination in terror related bomb explosion victims, safely decontaminating the victims while minimizing staff exposure, and allowing prompt care of both conventional and radiation related injuries. A successful response also requires designated radiation detection and monitoring equipment, and vigorous training of a large proportion of the hospital's staff.

Prehosp Disaster Med 2017;32(Suppl. 1):s2-s3

doi:10.1017/S1049023X17000358

\section{A Cost-effective Prescription for Radiological Emergency Preparedness in Community Hospital Emergency Departments}

\section{David Jaslow, E. S. Courtleigh, Derek Zecher}

Emergency Medicine, Lower Bucks Hospital, Bristol/PA/United States of America

Study/Objective: To present a cost-effective approach for community hospital emergency department (CH-ED) personnel, trained at the first receiver operations level, to deploy a radiation screening, detection, and decontamination capability. Background: Few $\mathrm{CH}-\mathrm{ED}$ are prepared to treat victims with external radiation contamination who might be seen after a terrorist attack using a radiological dispersal device. Furthermore, hospital staff or facilities may become secondarily contaminated if such victims are not identified and decontaminated immediately upon arrival. Demonstration of five actionable objectives defines $\mathrm{CH}-\mathrm{ED}$ hazmat/WMD preparedness: recognition/identification, notification, isolation, protection, and decontamination.

Methods: An operational system description which includes education, technical training, technology acquisition, and hazard-specific strategy and tactics is presented.

Results: Recognition (detection) requires a radiation area monitor $(\$ 6,000)$ to alert CH-ED staff that external contamination exists, prior to patients entering the treatment area. Staff then activate the emergency operations plan, notify the authority handling jurisdiction emergency services, and initiate the hospital incident command system. Hospital emergency response team members protect themselves by donning appropriate PPE (universal precautions) commonly used in
$\mathrm{CH}-\mathrm{ED}$. Contaminated patients are isolated in the decontamination room or placed into a decontamination corridor and individually scanned for the exact location, type, and severity (current dose rate) of radiological contamination; using a handheld pancake-type survey meter ( $\$ 600$ each x 2 ) by mid-level providers (MLPs) who have completed the Advanced Hazmat Life Support course ( $\$ 500$ each $\times$ 5). Decontamination is performed by nurses who have received in-house training using basic equipment and supplies which already exist in the decontamination room. Sustainment costs focus on educational needs and drills.

Conclusion: CH-ED capability to screen, detect, and decontaminate patients externally contaminated by radiation can be implemented for as little as 10 thousand US dollars and can be sustained for a fraction of the start-up cost.

Prehosp Disaster Med 2017;32(Suppl. 1):s3

doi:10.1017/S1049023X1700036X

\section{Comprehensive Disaster Medical System to Threat of Nuclear Emergency and Disaster}

Soon-Joo Wang

Hallym University, Hwaseong/Republic of Korea

Study/Objective: To develop a new comprehensive disaster medical system.

Background: The Government has developed the National Radiation EMS.

Methods: EMS for nuclear disasters were reviewed and re-organized.

Results: The primary emergency medical system around the nuclear plants was considered to be good, but there are problems during nights or holidays, for severe injury, and for many injured victims. The systems for decontamination in receiving facilities were not prepared enough. Personal protection devices for medical teams are less equipped. So, the new system is based on the assumption of nuclear disaster anywhere, any situation. It includes scenario of urban radiological material leakage, nuclear contamination from neighboring region, and mass panic state after perception of nuclear threat. National Radiation EMS developed a survey, an evaluation index of infrastructure, a prediction program for medical demand according to radiation disaster scenarios, and development plans. Evaluation indicators were composed of the seven domains: on-site response, ER, psychiatric support, radiation burn, bone marrow transplantation, internal contamination, and acute radiation syndrome. Each domain was measured by six grade levels. If 1,000 patients occur in the situation of combined disasters, according to the simulation analysis, the medical demand exceeds the capacity of the national radiation emergency medical response system. If 250 patients occur in case of a radioactivity leakage accident, it is expected to have some difficulty within the capacity of the regional response system, but it would be possible to respond within the national level.

Conclusion: The current level can be evaluated by comprehensive indicators and it is possible to plan the further development. For the adequate response to newly emerging threat of 